'0 Altun Koroglu, 'M Yalaz, ${ }^{2}$ E Levent, 'M Akisu, 'N Kultursay. ' Neonatology; ${ }^{2}$ Pediatric Cardiology, Ege University Faculty of Medicine, Izmir, Turkey

Backgroud and aims: Bronchopulmonary dysplasia (BPD) is one of the most important chronic complications of premature birth. Although long term effects of BPD are more commonly known by the well-defined pulmonary consequences, cardiovascular sequelae related to BPD have also been reported. In the post-surfactant era data on the cardiovascular changes in new BPD patients is limited. In this study we aimed to investigate the role of myocardial tissue Doppler echocardiography in detecting cardiac pathology in preschool BPD patients and to find out possible risk factors related to cardiovascular sequela.

Methods Prematurely born children with $\mathrm{BPD}(\mathrm{N}=21,4$ severe $\mathrm{BPD}, 3$ moderate BPD and 14 mild BPD) and without BPD (N=20) at 2 to 4 years of age were enrolled to the study. Conventional and myocardial tissue Doppler echocardiography studies were performed.

Results In conventional echocardiography; right ventricular fractional shortening, tricuspid E/A ratio, mitral late diastolic inflow velocity and pulmonary acceleration time were decreased; mitral $\mathrm{E} / \mathrm{A}$ ratio, left and right ventricular myocardial performance indexes were increased in BPD group compared to controls. In myocardial tissue Doppler measurements; tricuspid annulus E'/A'ratio was decreased and interventricular septum systolic velocity was increased in BPD group. Low birth weight, disease severity and postnatal cumulative steroid dosage were related with echocardiographic changes.

Conclusion BPD affects global cardiac performances up to 2 to 4 year of age with regard to birth weight, disease severity and cumulative steroid dosage. Myocardial tissue Doppler examination did not have additional value to conventional echocardiography in demonstration of these changes.

\section{EVALUATION OF INFLAMATION IN BRONCHOPULMONARY DISEASE WITH TRANSCUTANEOUS CARBOXIHEMOGLOBIN MEASUREMENT- PRELIMINARY RESULTS}

doi:10.1136/archdischild-2012-302724.0600

D Gonulal, M Yalaz, D Terek, 0 Altun Koroglu, 0 Uygur, N Kultursay. Neonatology, Ege University Faculty of Medicine, Izmir, Turkey

Background and Aims Bronchopulmonary dysplasia (BPD) is an important chronic respiratory morbidity of premature infants. Increased carboxyhemoglobin $(\mathrm{COHb})$ levels have been reported for chronic obstructive pulmonary disease, systemic inflammatory response syndrome and acute respiratory distress syndrome and also for mortality in premature infants. $\mathrm{COHb}$ levels increases as a result of oxidative stress and inflammation. Changes of $\mathrm{COHb}$ levels by the measurement of transcutaneous $\mathrm{COHb}$ levels may be informative for continuing inflammation levels of BPD. We aimed to evaluate inflammatory process in BPD with transcutaneous $\mathrm{COHb}$

Methods Twenty premature infants discharged from Ege University NICU with the diagnosis of BPD (Group 1), 20 premature infant without BPD (Group 2), 20 term healthy control (Group 3) infant were included in the study. Transcutanous $\mathrm{COHb}$ levels were measured with Masimo radical set device following three months after discharge. Antenatal and neonatal characteristics of infants were recorded.

Results Mean transcutanous $\mathrm{COHb}$ levels were significantly higher in group 1 than group $2(p=0.000)$ at postnatal age 0 . Mean $\mathrm{SpCO}$ values after first three months of discharge were higher in group 1 than group $2(p<0.05)$ and group $3(p<0.001)$. No difference was detected in the same groups' (Group 1 and 2 ) consequent measurements of SpCO.
Conclusion Our data support the ongoing persistant chronic inflamatory process after discharge in infants with BPD. The long term multisystemic morbidity, inflammatory mass could be minimized with early diagnosis and preventive treatments. Further investigations are needed in larger populations for early prediction of BPD among the risk group.

\section{DOES BRONCHOPULMONARY DYSPLASIA RELATE TO REDOX STATUS IN INFANTS LESS THAN 29 WEEKS OF GESTATIONAL AGE?}

doi:10.1136/archdischild-2012-302724.0601

${ }^{1}$ ISI Mohamed, ${ }^{2,3} \mathrm{JC}$ Lavoie. ${ }^{1}$ Pediatrics - Neonatology; ${ }^{2}$ Pediatrics- Neonatology; ${ }^{3}$ Centre de Recherche, Universite de Montreal - CHU Sainte-Justine, Montréal, OC, Canada

Background Glutathione is the key molecule in detoxification of peroxides leading to an oxidized glutathione redox status (GRS). We hypothesizes that GRS plays an important role in the etiology of bronchopulmonary dysplasia (BPD).

Objective To test the relation between GRS at 6-7 days of life as well as at 36 weeks of corrected age and BPD. To identifty perinatal factors affecting GRS.

Design/methods Whole blood GRS was measured at 6-7 days of life and at 36 weeks of corrected age (CA) in 51 infants less than 29 weeks of gestational age (GA). Perinatal clinical data that may affect the GRS were colleted. The GRS was calcualted using concentration of GSH and GSSG according to the Nernst equation (Schafer \& Buettner, 2001).

Results Infants in our cohort had gestational age of $26 \pm 1$ weeks with birth weight of $847 \pm 166 \mathrm{gm}$. Significant relation between GRS and BPD was confirmed with less risk of BPD in infants with most reduced GRS (day 6-7) and higher risk of BPD for infants with most oxidized GRS at 36 weeks CA.

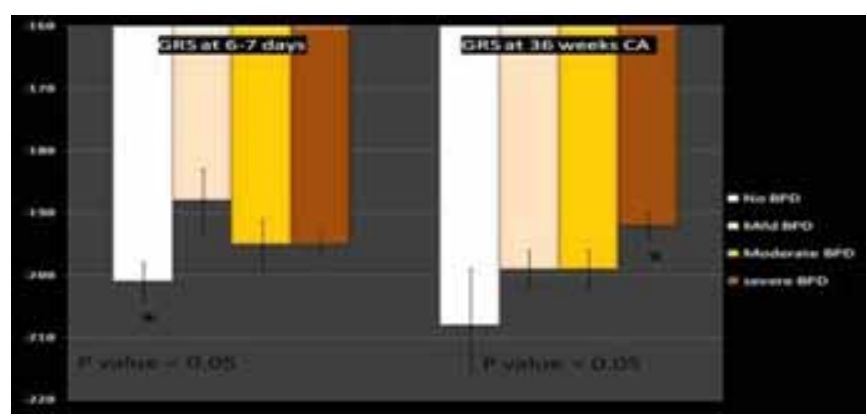

Abstract 601 Figure 1 Relation between GRS and BPD

GA and BW were signifcantly related to GRS

Abstract 601 Table 1 Different perinatal factors effect on GSR

\begin{tabular}{llll}
\hline & Test used & $\begin{array}{l}\text { P value for GRS } \\
\text { at 6-7 days }\end{array}$ & $\begin{array}{l}\text { P value } \\
\text { at 36 CA }\end{array}$ \\
\hline Gestational age & Pearson correlation & 0.01 & 0.08 \\
Birth weight & Pearson correlation & 0.04 & 0.93 \\
Sex & T-test & 0.47 & 0.89 \\
Maternal preeclampsia & T-test & 0.37 & 0.35 \\
Chorioamnionitis & T-test & 0.15 & 0.98 \\
Small for gestational age & T-test & 0.8 & 0.77 \\
\hline
\end{tabular}

Conclusions There is a significant relation between GRS 6-7 days of life as well as at 36 weeks CA and BPD outcome in infants less than 29 weeks of GA. The significant impact of both GA and BW on GRS at 6-7 days of life is explained by the glutathione level that is correlated with gestational age. 


\section{INCREASED INCIDENCE OF BRONCHOPULMONARY DYSPLASIA IN PRETERM INFANTS EXPOSED TO MATERNAL PREECLAMPSIA}

doi:10.1136/archdischild-2012-302724.0602

H Ozkan, M Cetinkaya, N Koksal. Uludağ University Medical Faculty, Bursa, Turkey

Objective The aims of the study were to determine the effect of preeclampsia on bronchopulmonary dysplasia (BPD) development in preterm infants and to investigate the possible association between BPD severity and preeclampsia.

Methods The study group involved preterm infants ( $\leq 32$ gestational week) born to a preeclamptic mother with no co-existing medical condition, whereas the comparison group involved preterm infants born to a normotensive mother. BPD was defined as requirement for supplemental oxygen for the first 28 day of life. It was classified as mild, moderate and severe BPD. The demographics and clinical data of the infants were recorded.

Results There were 117 and 215 premature infants in the study and control groups, respectively. The incidence of BPD in preterm infants in the study group (38.5\%) was significantly higher than the control group (19.5\%). The frequency of moderate and severe BPD were significantly higher in the study group. In logistic regression model, preeclampsia was found to be predictive of BPD.

Conclusions Preeclampsia was found to be an important risk factor for BPD development in preterm infants. The incidence of both moderate and severe BPD was significantly higher in preeclamptic mother infants. These findings might be associated with altered angiogenesis in the preeclamptic mother which might be shared with the fetus.

\section{OXYGEN DEPENDENCE IS ASSOCIATED WITH SUPPLY PROTEIN OFFERS AT FIRST 35 DAYS OF LIFE FOR PRETERM INFANTS WITH BRONCHOPULMONARY DYSPLASIA}

doi:10.1136/archdischild-2012-302724.0603

A Moraes-Barbosa, H Pacheco. Pediatrics, Federal Fluminense University, Niteroi, Brazil

Background and Aims The aims of this study was to determine if the time of introduction and diary quantity of amino acid offered at first 35 days of life for newborns with BPD, weight $<1500 \mathrm{~g}$ at birth, may influence the oxygen dependence at final period.

Methods Newborns with BPD, consecutively observed in the NICU (UFF - RJ - Brazil), from April 2006 to March 2008, were divided in Early Start (ES) and Late Start (LE) Groups when they began to received amino acids (AA) up $(n=56)$ or after $(n=48) 72$ hours of life. It was also divided according to the quantity of AA administered in the first week of life $(1.11 \pm 0.04 \mathrm{~g} / \mathrm{kg} /$ day): Larger Supply (LAS) $(n=42)$ and Lower Supply (LOS) $(n=58)$ received a quantity of AA 2 SD above or below this mean.

Results A total of 104 newborns were evaluated. At $35^{\text {th }}$ of life the babies of ES and LE were weighing $34.1 \%$ and $21.9 \%$ more than his weight at birth, respectively, and from LOS and LAS, 39\% and $23.7 \%$ regarding his birth ( $p>0.05)$. At the end of the studied period, $8.9 \%$ of the babies of the ES and $16.7 \%$ of the LE still stayed receiving some oxygen. In LAS $4.7 \%$ and $21 \%$ in LOS were receiving some oxygen support $(p=0.004)$.

Conclusion Nutritional state is decisive in the oxygen necessity at neonatal final period, and the major offers of protein is a priority way.

604 URINARY NGAL (UNGAL) AT BIRTH IS RELATED TO
BRONCHOPULMONARY DYSPLASIA IN PRETERM INFANTS
'S Vandini, 'G Aquilano, ${ }^{2} \mathrm{C}$ Capelli, ' $\mathrm{LT}$ Corvaglia, 'S Galletti, ${ }^{2} \mathrm{G}$ La Manna. 'Neonatology S. Orsola-Malpighi Hospital; ' $N$ ephrology S. Orsola-Malpighi Hospital, University of Bologna, Bologna, Italy

Background and Aims Bronchopulmonary dysplasia (BPD) is a chronic lung disease associated with premature birth and early lung injury. The pathogenesis is multifactorial, including fluid and electrolytes balance that is dependent to renal development during the first weeks of life.

We previously found a correlation between renal development during the first weeks of life and urinary neutrophil gelatinase-associated lipocalin (UNGAL) at birth in very low birth weight infants (VLBW). The aim of this study was to examine the relationship between urinary (UNGAL) and serum NGAL (SNGAL) at birth and BPD.

Methods UNGAL and SNGAL were determined at birth in VLBW. BPD was defined as oxygen need at 36 week gestational age (GA). Statistical analysis was performed with chi square.

Results 44 VLBW admitted at birth in our NICU were included in the study; 2 of them died during stay in NICU. 20/42 infants developed BPD: all were born at $\leq 29$ week (GA) and 14 of them needed diuretics. High values of UNGAL (>100 ng/ml) were observed more frequently among BPD treated with diuretics infants than in the other subjects $(57 \%$ vs $28 \%$, p=0.04).

High levels of SNGAL (>150 ng/ml) were not significantly more frequent in VLBW with BPD.

Conclusions These preliminary data show that high UNGAL at birth is a marker of impaired renal development and fluid balance in preterm newborns, that determine increased lung water and consequently contribute to BPD development.

\section{5 "LOW DOSE" POSTNATAL CORTICOSTEROIDS FOR INFANTS AT RISK OF SEVERE BRONCHOPULMONARY DYSPLASIA}

doi:10.1136/archdischild-2012-302724.0605

${ }^{1,2}$ C Pitan. 'Neonatology, Royal Women's Hospital, Melbourne, VIC, Australia; 2Paediatrics, University College Dublin, Dublin, Ireland

Background Postnatal corticosteroids (PCS) improve short term lung function but may increase the risk of disability in later life. The Dexamethasone: A Randomised Trial (DART) study was designed to address this risk using a 10 day tapering regimen with a total dose of 890 micrograms $/ \mathrm{kg}$. The Royal Women's Hospital, Melbourne selects patients at high risk of bronchopulmonary dysplasia (BPD) to receive steroids as per the DART protocol. The primary aim of this study was to audit the prescribing practices of the DART protocol with a secondary aims of reporting patient outcomes.

Method Patients were identified from an electronic database between January 2006 and December 2009 and medical records reviewed. Infants prescribed PCS other than as per the DART protocol and for preventing BPD were excluded.

Results Forty six infants with mean (SD) gestational age of 25.0 (1.3) weeks and birth weight of 685 (192) g were prescribed dexamethasone. Median (range) duration of therapy was 20 (3-86) days with a median total dexamethasone dose of 1437 (375-9100) micrograms $/ \mathrm{kg}$. There were significant changes on day 3 of treatment in mean airway pressure (MAP) or positive end expiratory pressure (PEEP), $\mathrm{p}<0.001$ and percentage inspired oxygen $\left(\mathrm{FiO}_{2}\right), \mathrm{p}<0.001$. There were seven deaths, with the combined outcome of either death or BPD occurring in 41 (89\%) infants.

Conclusions In our audit prescribing practices deviating outside of hospital protocol, resulting in doses exceeding those recommended. Clinical practice review of PCS therapy in preterm infants is encouraged.

606 DIURETIC USE IN NEONATAL CHRONIC LUNG DISEASE IN ENGLAND 